Parepa Irinel-Raluca, Suceveanu Andra-Iulia, Suceveanu A. P., Mazilu Laura, Cîmpineanu B., Craiu Elvira

\title{
Upper endoscopy in patients with acute ST-elevation myocardial infarction [STEMI] and postthrombolysis upper gastrointestinal bleeding - results, therapeutic utility
}

2nd Department, Medical Sciences, Faculty of Medicine, “Ovidius” University of Constanţa

\begin{abstract}
Upper endoscopy is the "golden standard" for the diagnosis of upper gastrointestinal bleeding (UGB); it appreciates the persistence of bleeding in more than $90 \%$ of cases, it reveals the UGB lesion, gives prognostic information about re-bleeding risk and offers the possibility of endoscopic haemostasis. Uncomplicated STEMI by itself is not a contraindication for upper endoscopy $[1,2]$. The aim of our study was to observe the haemorrhagic lesions in STEMI patients with postthrombolysis UGB and to evaluate the safety and therapeutic utility of upper endoscopy in this category of patients. We performed upper endoscopy in STEMI patients with postthrombolytic UGB during a period of 4 years (1st of Jan $2008-31$ st of Dec 2011). Patients with systolic blood pressure (SBP) $<100 \mathrm{mmHg}$, with Killip class $>1$, with unstable ECG and/or with severe associated conditions (respiratory distress, cerebrovascular stroke, consciousness disorders) were withdrawn from undergoing upper endoscopy, as well as patients who refused the investigation. During studied period, 618 STEMI patients underwent pharmacological coronary reperfusion. Postthrombolysis UGB occurred in 42 of them $(6.79 \%)$. We could perform upper endoscopy in 30 patients $(71.43 \%)$, the rest of $12(28.57 \%)$ being withdrawn from endoscopy (6 patients with Killip class $>1,4$ patients with systolic blood pressure $<100 \mathrm{mmHg}, 2$ patients refused the investigation). Incriminated haemorrhagic lesions
\end{abstract}

Parepa Irinel-Raluca

2nd Department, Medical Sciences

Faculty of Medicine, "Ovidius” University of Constanţa

Constanţa County Emergency Hospital

145 Tomis Blvd, Constanta

e-mail: irinel_parepa@yahoo.com

Tel./Fax: +40-241-553022 were: erosive gastroduodenitis in 20 cases $(66.67 \%)$ and duodenal ulcer in 10 cases $(33.33 \%)$. As about therapeutic utility, amongst 30 patients investigated just 4 needed endoscopic treatment (haemoclips used in Forrest IB patients with duodenal ulcers). Endoscopy was a safe investigation in STEMI patients with postthrombolysis UGB. Erosive gastroduodenitis was incriminated as the source of bleeding in most of the cases. As it concerns the therapeutic utility of UE in STEMI patients with UGB, $13.33 \%$ of bleeding patients benefited from endoscopic treatment (mechanical haemmostasis).

Keywords: upper endoscopy, acute STEMI, postthrombolysis upper gastrointestinal bleeding.

\section{Background}

Pharmacologic thrombolysis is used as an emergency therapy for obtaining coronary reperfusion immediately after an acute STEMI (within 6 hours from STEMI onset). Until very recently (July 2012), pharmacologic thrombolysis was the only coronary reperfusion therapy used in Constanţa County for STEMI patients. One of the major, life-threatening complications after thrombolysis is overt upper gastrointestinal bleeding (UGB), exteriorised by hematemesis and/or melena. The "golden standard" for the diagnosis of UGB is upper endoscopy.

Upper endoscopy is a common examination, with a low rate of complications. The global 
mortality rate is less than $0.1 \%$ [3]. Most common complications during upper endoscopy are represented by cardiovascular events, due to the underlying heart diseases [4]. Despite of this fact, most literature data emphasize that stable acute/recent STEMI by itself is not o contraindication for upper endoscopy $[1,2,5,6]$.

\section{Aim of the study}

Starting from above presented data and from the facts that upper endoscopy reveals the UGB lesions, appreciates the persistence of bleeding in more than $90 \%$ of cases and offers the possibility of endoscopic hemostasis - we aimed to evaluate the safety and therapeutic utility of upper endoscopy in STEMI patients with postthrombolysis UGB.

\section{Material and methods}

We performed a prospective study over a period of 4 years (1st of Jan 2008 - 31st of Dec 2011). Inclusion criteria were: patients with acute but stable STEMI who underwent thrombolysis complicated by overt UGB (hematemesis and/or melena). Exclusion criteria were: patients with acute STEMI and postthrombolysis UGB and with systolic blood pressure $(\mathrm{SBP})<100 \mathrm{mmHg}$, with Killip class $>1$, with unstable ECG and/or with severe associated conditions (respiratory distress, cerebrovascular stroke, consciousness disorders), as well as patients who refused the upper endoscopy. Patients performed upper endoscopy in the Endoscopy unit of Gastroenterology Department of Constanţa County Hospital, closely supervised by a mixed team of cardiology - gastroenterology - intensive care specialists.

\section{Results}

During studied period, 618 STEMI patients underwent pharmacological coronary reperfusion. Postthrombolysis UGB occurred in 42 of them $(6.79 \%)$ (Figure 1).

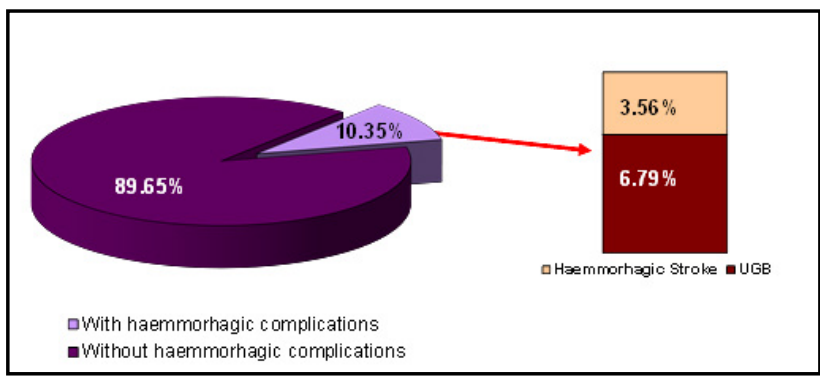

Figure 1.Global incidence of major bleeding complications in patients with STEMIwho underwent thrombolysis

We could perform upper endoscopy (UE) in 30 patients $(71.43 \%)$, the rest of $12(28.57 \%)$ being withdrawn from endoscopy (6 patients with Killip class $>1,4$ patients with systolic blood pressure $<100 \mathrm{mmHg}, 2$ patients refused the investigation) (Figure 2).

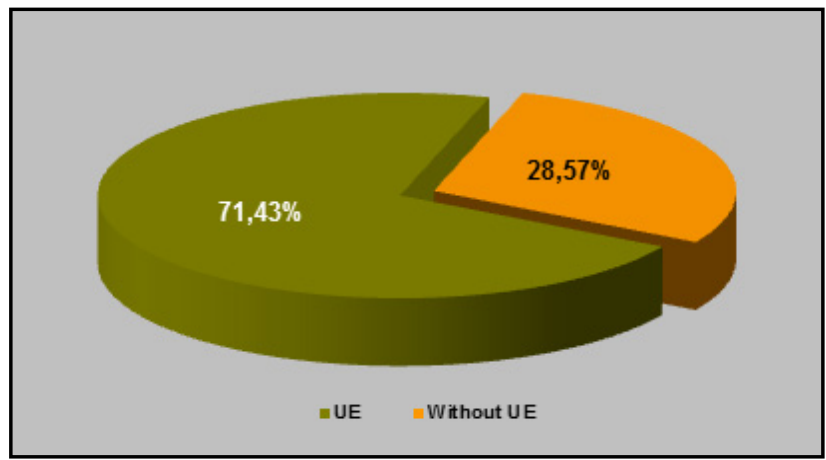

Figure 2.\% patients with STEMI and UGB who underwent $U E$

Neither of the investigated patients had any complication or unwanted event during or after UE.

Hemmorhagic lesions identified in STEMI patients with postthrombolysis UGB who underwent 
UE were (figure 3):

- Erosive gastroduodenitis - 20 cases

- Duodenal ulcer - 10 cases

As it concerns the therapeutic utility of UE in STEMI patients with UGB, only 4 patients out of 30 needed endoscopic treatment $(13.33 \%)$. These patients presented Forrest IB gastroduodenal bleeding due to duodenal ulcers, and they benefited from endoscopic haemmostasis (mechanical haemmostasis by using haemmoclips) (Figures 3 - 5).

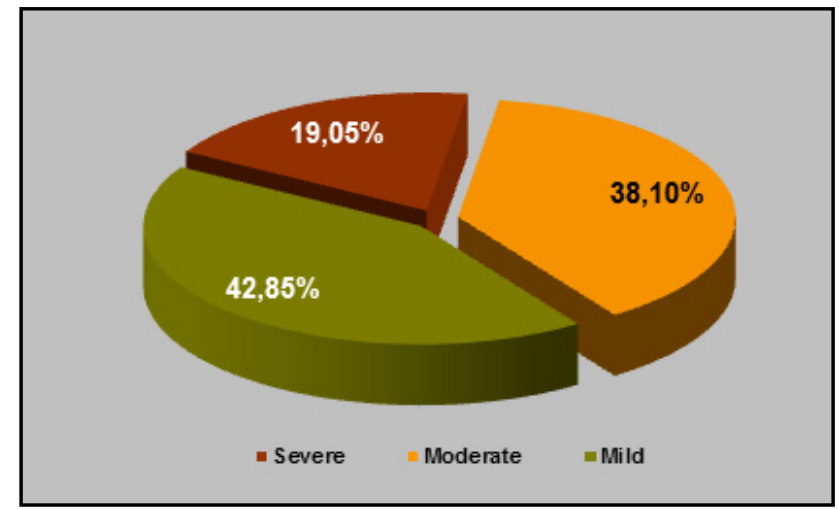

Figure 5. Severity of posthaemmorhagic anemia in STEMI patients with postthrombolysis $U G B(\%)$

UGB may be one of the major, life-threatening complications after thrombolysis. In our study it occurred in $6.79 \%$ of STEMI patients who underwent thrombolysis. Upper endoscopy is the "golden standard" for the diagnosis of UGB; it is a common examination, with a low rate of complications. Most studies showed that acute/recent STEMI by itself is not o contraindication for upper endoscopy if the patient is stable. In our study upper endoscopy could be performed in $71.43 \%$ of STEMI patients with postthrombolysis UGB, with no procedural complications, proving once again to be a safe investigation (Figure 6).

\section{Conclusions}

Endoscopy was a safe investigation in STEMI patients with postthrombolysis UGB. Erosive gastroduodenitis was incriminated as the source of bleeding in most of the cases. As it concerns the therapeutic utility of UE in STEMI patients with UGB, $13.33 \%$ of bleeding patients benefited from endoscopic treatment (mechanical haemmostasis).

Figure 4. Therapeutic utility of UE in STEMI patients with postthrombolysis $U G B(\%)$ 

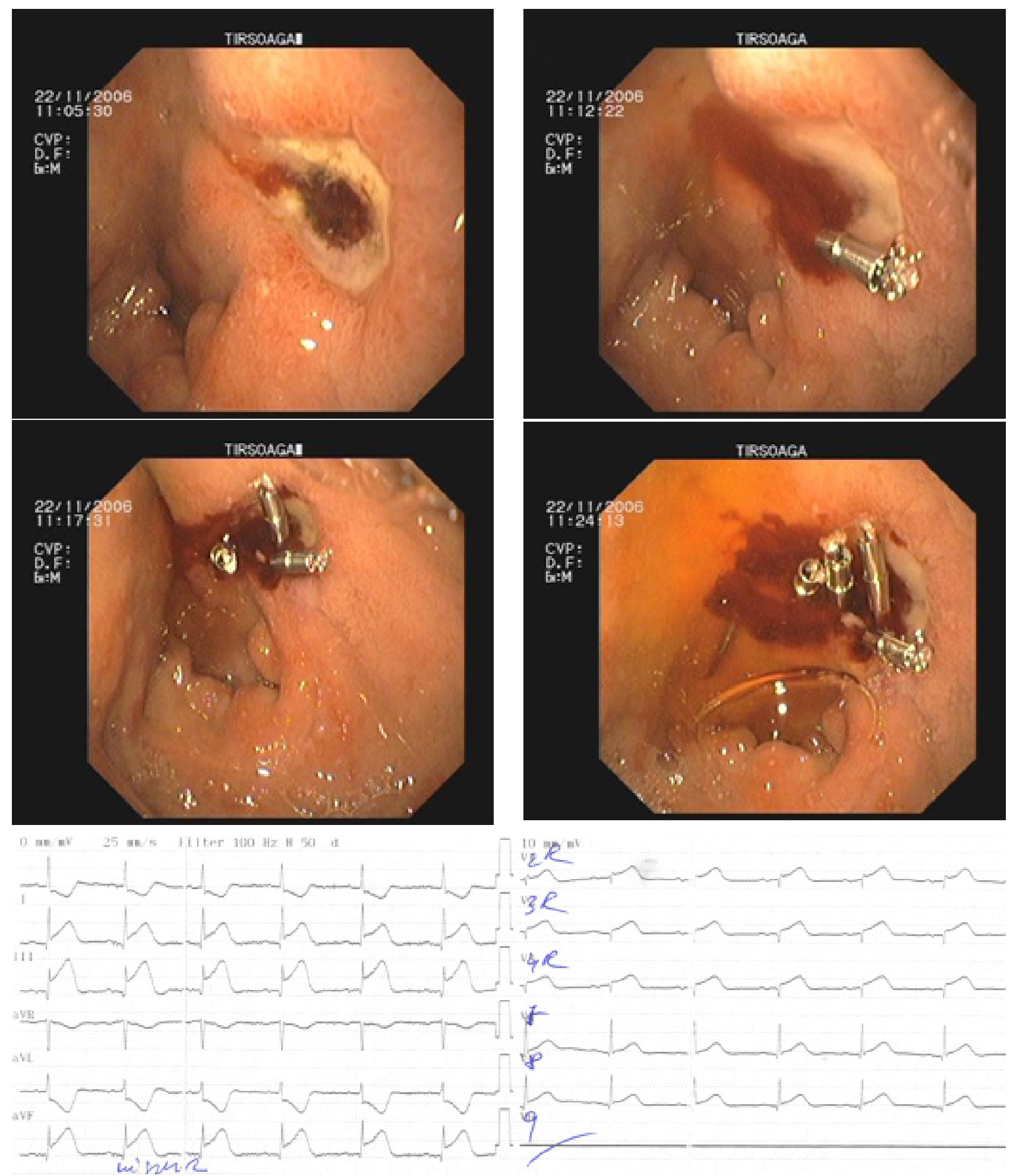

Figure 6. Forrest IB duodenal ulcer in a patient with postero-inferior and right ventricle STEMI (after thrombolysis). Endoscopic haemmostasis was obtained with 5 haemmoclips 


\section{References}

1. Mumtaz K. \& Ismail FW et al. (2008) Safety and utility of oesophago-gastro-duodenoscopy in acute myocardial infarction. Eur J Gastroenterol Hepatol. 20(1), 51-55.

2. Lin S, Konstance R, Jollis J. \& Fisher DA. (2006). The utility of upper endoscopy in patients with concomitant upper gastrointestinal bleeding and acute myocardial infarction. Dig Dis Sci. 51(12), 2377-2383

3. Cappell MS. (1996). Gastrointestinal endoscopy in high-risk patients. Dig Dis. 14(4), 228-244
4. Davis RE. \& Graham DY. (1979) Endoscopic complications. Gastrointestinal Endosc. 25, 146149.

5. Cappell MS. (1993.) The safety and clinical utility of esophagogastroduodenoscopy for acute gastrointestinal bleeding after myocardial infarction: a six-year study of 42 endoscopies in 34 consecutive patients at two university teaching hospitals. Am J Gastroenterol. 88 (3), 344-350

6. Cappell MS. \& Iacovone FM. (1999). Safety and efficacy of esophagogastroduodenoscopy after myocardial infarction. Am J Med. 106(1), 29-35. 